\title{
Caenorhabditis elegans caspase homolog CSP-2 inhibits CED-3 autoactivation and apoptosis in germ cells
}

\author{
X Geng ${ }^{1,4}$, QH Zhou ${ }^{1,4}$, E Kage-Nakadai ${ }^{2}$, Y Shi ${ }^{3}$, N Yan ${ }^{3}$, S Mitani ${ }^{2}$ and D Xue ${ }^{\star, 1}$
}

In Caenorhabditis elegans, apoptosis in germ cells is mediated by the same core apoptotic machinery that controls apoptosis in somatic cells. These include the CED-3 caspase, the CED-3 activator CED-4, and the cell death inhibitor CED-9. However, germline apoptosis also differs from somatic apoptosis in its regulation. We found that CSP-3, a caspase homolog that blocks CED-3 autoactivation and apoptosis in somatic cells, does not affect apoptosis in germ cells. Interestingly, the second C. elegans caspase homolog, CSP-2, shares sequence similarity to both catalytic subunits of the CED-3 caspase, and surprisingly, contains a stretch of sequence that is almost identical to that of CSP-3. Unlike CSP-3 that acts specifically in somatic cells, loss of CSP-2 causes increased apoptosis only in germ cells, suggesting that CSP-2 is a germ cell-specific apoptosis inhibitor. Moreover, like CSP-3, CSP-2 associates with the CED-3 zymogen and inhibits its autoactivation, but does not inhibit CED-4-induced CED-3 activation or the activity of the activated CED-3 protease. Thus, two different $C$. elegans caspase homologs use the same mechanism to prevent caspase autoactivation and apoptosis in different tissues, suggesting that this could be a generally applicable strategy for regulating caspase activation and apoptosis.

Cell Death and Differentiation (2009) 16, 1385-1394; doi:10.1038/cdd.2009.88; published online 3 July 2009

In Caenorhabditis elegans, many early developing germ cells undergo apoptosis during normal oogenesis; this process is called germline apoptosis. ${ }^{1,2}$ Germline apoptosis seems to be an integral component of the oogenesis program and has been suggested to be important for eliminating excess germ cells that acted as nurse cells to provide cytoplasmic components to maturing oocytes. ${ }^{1-3}$ Germline apoptosis is conserved among eukaryotes, from $C$. elegans to humans. Therefore, $C$. elegans provides an excellent model system for studying the regulation of germ cell apoptosis.

The molecular machinery that mediates germline apoptosis has been extensively studied and found to share key components with that of somatic apoptosis. In particular, CED-3, CED-4, and CED-9 are essential for apoptosis in both germ cells and somatic cells. ${ }^{1,2}$ However, regulation of apoptosis in germ cells and in somatic cells seems to differ significantly. For example, the pro-apoptotic $\mathrm{BH} 3$ only protein, EGL-1, is crucial for somatic apoptosis but is dispensable for physiological germ cell death. ${ }^{1}$ Moreover, $C$. elegans somatic cells are resistant to genotoxic insults, whereas germ cells readily respond to genotoxic stresses to undergo apoptosis, ${ }^{4}$ suggesting that the regulation of germ cell death could be fundamentally different from that of somatic cell death.

One crucial aspect of apoptosis regulation is the regulation of caspases, the aspartate-specific cysteine proteases that execute the cell killing process., ${ }^{5,6}$ Numerous caspase inhibitors have been identified and shown to inhibit the activation or the activities of caspases. In particular, the inhibitors of apoptosis proteins (IAPS), characterized by at least one baculoviral IAP repeat (BIR) and a RING finger motif, ${ }^{7}$ are conserved caspase inhibitors in Drosophila and higher organisms. ${ }^{8-12}$ However, no IAP homolog has been identified in $C$. elegans. Instead, a partial caspase homolog CSP-3 was found to protect $C$. elegans somatic cells from apoptosis by associating with the CED-3 zymogen and inhibiting CED-3 autoactivation. ${ }^{13}$ It is unclear whether CSP-3 also inhibits CED-3 autoactivation in C. elegans germ cells and how the activity of CED- 3 is negatively regulated in the germline.

In this study, we found that a second $C$. elegans caspase homolog, CSP-2, which shares sequence similarity to both catalytic subunits of CED-3 (Figure 1a), is expressed specifically in the $C$. elegans germline and acts as a germ-cellspecific cell death inhibitor. Moreover, CSP-2 associates with the CED-3 zymogen and specifically inhibits CED-3 autoactivation, but not CED-3 activation mediated by CED-4 or CED-3's catalytic activity. Our findings suggest that caspase homologs lacking protease activities serve as dedicated caspase inhibitors in $C$. elegans to prevent CED-3 from inadvertent autoactivation and cells from apoptosis.

\footnotetext{
${ }^{1}$ Department of Molecular, Cellular, and Developmental Biology, Campus Box 347, University of Colorado, Boulder, CO 80309, USA; ${ }^{2}$ Department of Physiology, Tokyo Women's Medical University, School of Medicine, and CREST, JST, 8-1, Kawada-cho, Shinjuku-ku, Tokyo 162-8666, Japan; ${ }^{3}$ Center for Structural Biology, Department of Biological Sciences and Biotechnology, Institute of Biomedicine, Tsinghua University, Beijing 100084, China

*Corresponding author: D Xue, Department of Molecular, Cellular, and Developmental Biology, University of Colorado, Campus Box 347, Boulder, CO 80309, USA. Tel: + 303492 0271; Fax: + 303492 7744; E-mail: ding.xue@ colorado.edu

${ }^{4}$ These authors contributed equally to this work.

Keywords: germ cell death; Caenorhabditis elegans; CED-3; caspase activation; cell death inhibitor

Abbreviations: AA, amino acid; GFP, green fluorescent protein; L1-L4, larval stages 1-4; N2, wild-type C. elegans strains-Bristol isolate; PCD, programmed cell death; RT-PCR, reverse transcriptase polymerase chain reaction; WT, wild type; CED, cell death abnormal; S.E.M, standard error of the mean

Received 18.3.09; revised 01.6.09; accepted 05.6.09; Edited by RA Knight; published online 03.7.09
} 

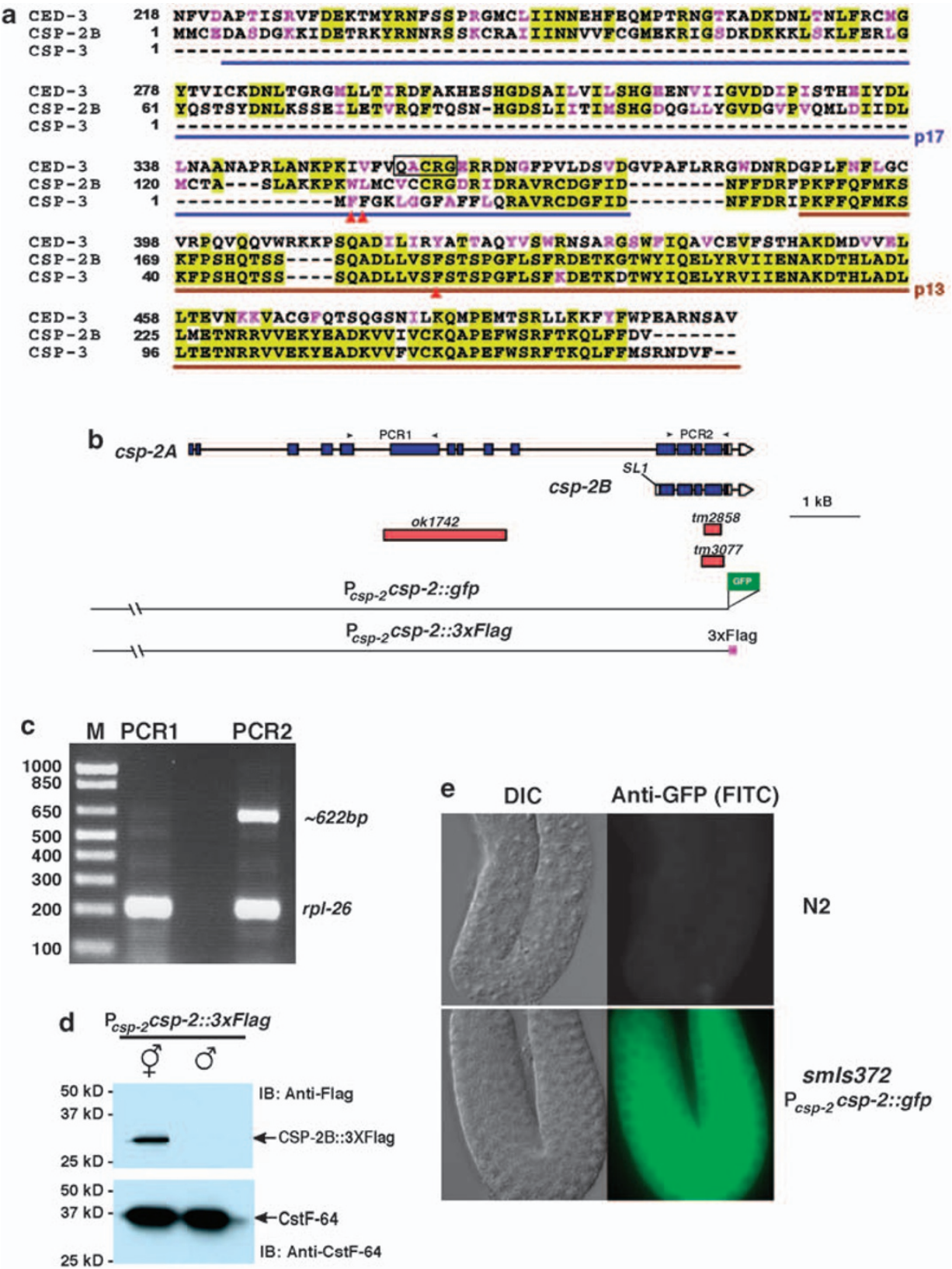

Figure 1 CSP-2, a CED-3 homolog, is expressed specifically in hermaphrodite germ cells. (a) Sequence alignment of CED-3, CSP-2B, and CSP-3. Residues that are identical are shaded in yellow and residues that are similar in pink. Trp131, Leu132, and Phe 186 of CSP-2B are indicated with red arrowheads. Underlines delineate the large and small subunits of CED-3, respectively. The box indicates the active-site pentapeptide of CED-3. (b) The csp-2 gene structure and deletion mutations. Exons are depicted as boxes and introns as lines. The translated regions of $c s p-2$ are highlighted in blue. Three red boxes indicate the regions of $c s p-2$ removed by the three $c s p-2$ deletions, respectively. SL1 stands for SL1 trans-spliced leader sequence. (c) RT-PCR analysis of the relative abundance of CSP-2A and CSP-2B transcripts. Reverse transcription was performed on poly(A)n mRNA isolated from mix-stage wild-type animals, followed by PCR amplification with primers specific to the CSP-2A coding region (PCR1 in $\mathbf{b}$ ), or primers in the CSP-2A/2B coding region (PCR2 in b), or primers specific for a control gene rpl-26 (encoding a large ribosomal subunit L26 protein). In the PCR1 lane, the predicted 706-bp CSP-2A-specific RT-PCR product was not seen. In the PCR2 lane, amplification of the 622-bp product corresponding to the CSP-2A/2B transcript was

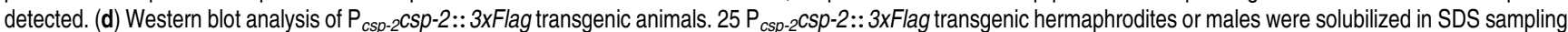
buffer and resolved on $15 \%$ SDS polyarylamide gel. The CstF-64 protein was used as a loading control. (e) $c s p-2$ is specifically expressed in $C$. elegans germ cells. Differential

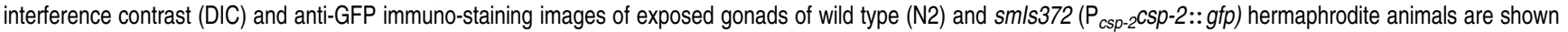

\section{Results}

Loss of csp-2 causes increased germ cell death but does not affect somatic cell death. CSP-2 was first identified as a caspase homolog that shares sequence similarity to both catalytic subunits of CED-3 (Figure 1a). However, CSP-2 does not seem to contain a caspase activity in vitro, ${ }^{14}$ probably because it lacks the invariant active-site pentapeptide QACXG (X could be $R, Q$, or $G$ ) found in all active caspases (VCCRG in CSP-2). ${ }^{15}$ Interestingly, the carboxyl terminal 
region of CSP-2 is homologous to the small subunit of CED-3 and shares $92 \%$ identity to CSP-3 (Figure 1a), raising an intriguing possibility that CSP-2 may act as a caspase inhibitor like CSP-3. To examine the function of csp-2 in C. elegans, we obtained three different csp-2 deletion alleles (Figure 1b). Two deletions, tm2858 and tm3077, remove parts of the carboxyl terminal region of CSP-2, which is present in both $c s p-2$ transcripts identified earlier (Figure 1b). ${ }^{14}$ These two $c s p-2$ transcripts conceptually encode two proteins, CSP-2A and CSP-2B, respectively. CSP-2B is identical to the carboxyl terminus of CSP-2A, which has an extra 563 amino acid at the amino terminus. As CSP-2B transcript is trans-spliced to the SL1 spliced leader, it is an independent, complete transcript. ${ }^{14,16}$ Indeed, RT-PCR analysis of mixed stage wild-type animals shows that CSP-2B is the dominant $c s p-2$ message and we barely detected any CSP-2A transcript (Figure 1c). Moreover, in western blot analysis, we only detected a protein of approximately $33 \mathrm{kDa}$, the size of CSP-2B, in worm lysate derived from animals carrying a complex transgene array expressing CSP-2::3xFlag under the control of its own promoter ( $\mathrm{P}_{c s p-2} c s p-2:: 3 x$ Flag; Figure $1 \mathrm{~b}$ and $\left.\mathrm{d}\right)$. These results indicate that $\mathrm{CSP}-2 \mathrm{~B}$ is the major $\mathrm{CSP}-2$ protein expressed in $C$. elegans, although very low-level expression of CSP-2A cannot be ruled out. The third deletion, ok1742, specifically removes a region in CSP-2A but does not affect the CSP-2B coding region.

To investigate whether csp-2 affects apoptosis, we counted the number of apoptotic cells in the germline and embryos of csp-2 deletion mutants, two developmental stages with active apoptosis events. As apoptotic cells are swiftly engulfed in $C$. elegans, we sensitized these cell corpse assays by counting ced-6(n2095), csp-2, and ced-2(n1994) csp-2 double mutants, in which cell corpse engulfment is disabled because of the inactivation of the ced-2 or the ced- 6 gene and a small increase in cell death will result in a greater increase in the number of persistent cell corpses. ${ }^{13,17}$ Unlike the csp-3(tm2260) mutation that causes increased cell deaths in $C$. elegans embryos and ectopic deaths of neurons that normally live, ${ }^{13} \operatorname{csp}-2$ (tm2858) and $\operatorname{csp}$-2(tm3077) do not seem to affect embryonic cell deaths (Figure 2a and b) or result in missing touch cells in larvae (Figure 2c; data not shown), suggesting that $c s p-2$ does not affect apoptosis in somatic tissues. However, we did observe a slight increase in germ cell deaths in csp-2(tm2858) and csp-2(tm3077) animals compared with wild-type animals (Figure $3 a$; data not shown) and the increase in germ cell corpses is easily detected in the ced-6(n2095) and ced-2(n1994) mutant backgrounds (Figure $3 \mathrm{~b}-\mathrm{e}$ ), suggesting that loss of csp-2 causes increased germ cell deaths and that CSP-2 inhibits apoptosis in germ cells. By contrast, we did not observe increased germ cell corpses in csp-3(tm2260), csp-3(tm2260); ced-6(n2095) or csp-3(tm2260); ced-2(n1994) animals, compared with wild-type, ced-6(n2095), and ced-2(n1994) animals, respectively (Figure $3 a, c$ and e). Nor did we observe further increase in germ cell deaths in the csp-3(tm2260); $\operatorname{csp}-2$ (tm3077) double mutant or in the csp-3(tm2260); ced-6(n2095); csp-2(tm3077) and csp-3(tm2260); ced2(n1994) csp-2(tm3077) triple mutants (Figure 3a, c, and e), indicating that $c s p-3$ does not contribute to the inhibition of germ cell death. Interestingly, unlike csp-2(tm2858) and csp2(tm3077) deletion mutations, csp-2(ok1742), which removes only the CSP-2A coding region, did not seem to affect germ cell death (Figure $3 \mathrm{c}$ ). This result is consistent with our observations that the CSP-2B transcript is the dominant $c s p-2$ transcript in $C$. elegans and suggests that CSP-2B is mainly responsible for inhibiting germ cell death in the csp-2 locus and that CSP-2A is not critical for this inhibitory function. We thus only analyzed the activity of CSP-2B hereafter.

Loss of csp-2 reduces animal brood size. We also examined whether ectopic deaths of germ cells in csp-2(If) mutants affect brood size, as has been observed in animals deficient in ced-9, another anti-apoptosis gene. ${ }^{18}$ Both csp-2 mutants (tm3077 and tm2858) had significantly reduced brood sizes, approximately $60 \%$ of that observed in wild-type animals (Table 1). On the other hand, the csp-2(ok1742) mutant, which did not display ectopic germ cell deaths, had a normal brood size (Figure 3c). Moreover, csp-2(tm3077) exacerbated the sterility defect caused by a weak loss-offunction mutation in ced-9 (n1653ts), ${ }^{18}$ resulting in complete sterility of the ced-9(n1653ts); csp-2(tm3077) double mutant (Table 1). These results suggest that $c s p-2$ is important for normal germline development in $C$. elegans.

CSP-2B is specifically expressed in $C$. elegans germline. We next examined the expression patterns of $c s p-2$ by constructing a CSP-2 translational GFP fusion under the control of its own promoter $\left(\mathrm{P}_{c s p-2} c s p-2:: g f p\right)$ and generating a low-copy integrated transgene (sm/s372) carrying $\mathrm{P}_{s p-2} c s p-2:$ :gfp through biolistic bombardment (Figure 1b). ${ }^{19}$ sm/s372 fully rescued the increased germ cell corpse phenotype of the ced-2(n1994) csp-2(tm3077) mutant (Figure $3 \mathrm{~g}$ ), suggesting that the fusion protein is expressed in the right cells and targeted to the appropriate cellular locations. Using an antibody to GFP, we detected CSP-2::GFP in the cytoplasm of all germ cells of sm/s372 hermaphrodite animals but failed to see any GFP staining in somatic cells (Figure 1e; data not shown). This cytoplasmic staining pattern of CSP-2::GFP is similar to that of CSP$3::$ GFP ${ }^{13}$ suggesting that both proteins act in cytoplasm to inhibit apoptosis. Interestingly, in immunoblotting analysis of worm lysate from male or hermaphrodite animals carrying the $\mathrm{P}_{c s p-2}$ csp-2:: 3xFlag complex array, we detected expression of CSP-2B only in hermaphrodites but not in males (Figure 1d). As no cell death occurs in male germline, ${ }^{1,2}$ expression of CSP-2 may be dispensable in males.

To verify that germline expression of CSP-2B is sufficient to mediate the $\operatorname{csp}-2$ function, we expressed a GFP::CSP-2B fusion under the control of the pie-1 promoter $\left(\mathrm{P}_{\text {pie-1 }} \mathrm{GFP}:\right.$ : CSP-2B) in complex transgene arrays that allow expression of transgenes in the germline. ${ }^{20} \mathrm{P}_{\text {pie-1 }} \mathrm{GFP}:$ :CSP-2B fully rescued the increased germ cell corpse phenotype of the ced-6(n2095); csp-2(tm3077) mutant, whereas a mutant $\mathrm{P}_{\text {pie-1 }} \mathrm{GFP}:$ : CSP-2B(W131E, L132R, F186D) construct failed to do so (Figure 3f; see below), confirming that CSP-2B is responsible for the csp-2 activity in inhibiting germ cell deaths. We could also rescue the csp-2(If) defect or even cause mild suppression of germ cell deaths by ubiquitously overexpressing $\mathrm{CSP}-2 \mathrm{~B}$ under the control of the 

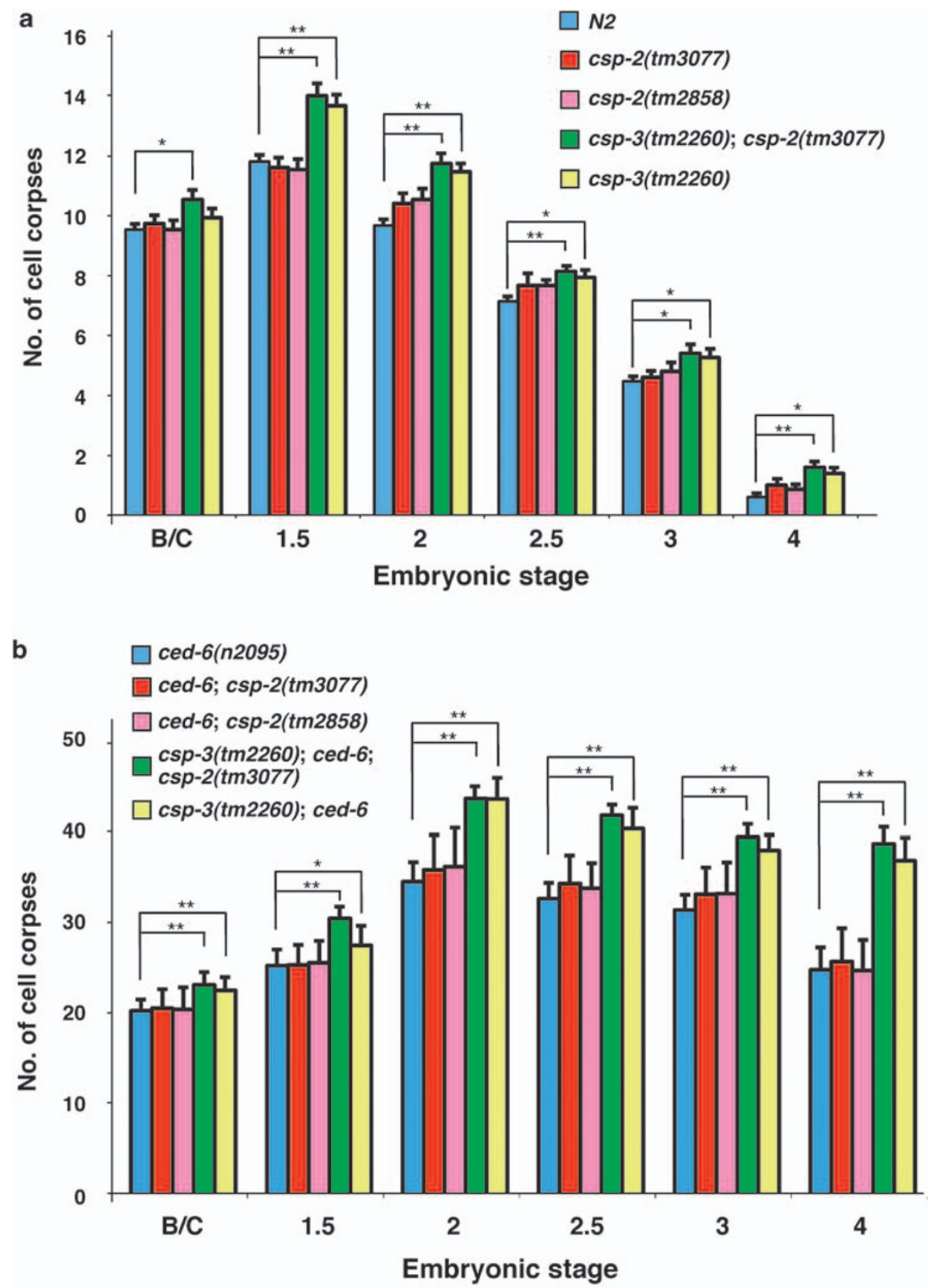

c

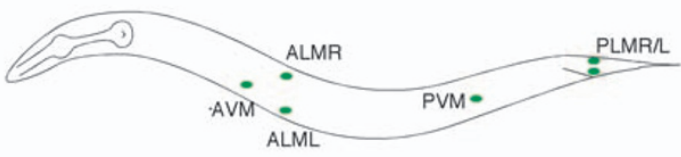

\begin{tabular}{lc} 
Genotype & $\begin{array}{c}\% \text { animals missing at } \\
\text { least one touch cell }\end{array}$ \\
\hline bzls8 & $1.0 \%$ \\
$c s p-3($ tm2260); bzls8 & $30.0 \%$ \\
$c s p-2(t m 3077) ; b z l s 8$ & $2.0 \%$ \\
$c s p-3(t m 2260) ; c s p-2(t m 3077) ; b z l s 8$ & $33.0 \%$ \\
\hline
\end{tabular}


heat-inducible promoter $\left(\mathrm{P}_{h s p} c s p-2 B\right)$ from a low-copy transgene (sm/s389) generated by biolistic bombardment (Figure $3 \mathrm{~g}$ ). Moreover, the $\mathrm{P}_{h s p} c s p-2 B$ transgenes could rescue the missing cell defect of the csp-3(If) mutant in somatic cells (Figure $3 \mathrm{~h}$ ). ${ }^{13}$ However, like CSP $-3,{ }^{13}$ overexpression of CSP-2B in soma did not obviously suppress the death of somatic cells that are programmed to die (data not shown). These results indicate that $c s p-2$ may use a mechanism like that of $c s p-3$ to prevent cell death.

CSP-2B associates with the CED-3 zymogen. As CSP-2B shares sequence similarity to both the large and the small subunits of CED-3 that form the active p17/p13 heterodimeric protease complex (Figure 1a), ${ }^{21}$ we tested whether CSP-2B may associate with CED-3 in vitro like CSP-313. ${ }^{13}$ Using a glutathione $S$-transferase (GST) fusion protein pull-down assay, the CED-3 zymogen tagged with a Flag epitode was pulled down by the GST-CSP-2B fusion, when both were co-expressed in bacteria (Figure 4a). By contrast, the GST control protein failed to pull down CED-3, suggesting that CSP-2 interacts with the CED-3 zymogen. We then characterized the interaction between CSP-2B and different domains of CED-3 and found that the large subunit (p17) and the small subunit (p13) of CED-3 each could associate with CSP-2B specifically (Figure 4b). Therefore, CSP-2B may interfere with the function of CED-3 by binding to either region of the CED-3 zymogen.

To identify interface residues that are important for CSP-2B binding to CED-3, we constructed a three-dimensional structural model of the CED-3/CSP-2 complex based on the crystal structure of active caspase-3 (Figure $4 c$ ). ${ }^{22}$ We then mutated several potential interface residues and examined whether these mutations interfered with the binding of CSP2B to CED-3 (data not shown). We found that Trp131 and Leu132, two residues in a CSP-2B region homologous to the large subunit of CED-3, were required for CSP-2B binding to the small subunit of CED-3 (Figure 1a; bottom panel of Figure 4b). On the other hand, Phe186 situated in a CSP-2B region homologous to the small subunit of $C E D-3$ was important for CSP-2B binding to the large subunit of CED-3 in vitro (Figure 1a; the upper panel of Figure $4 \mathrm{~b}$ ). Moreover, a CSP-2B mutant carrying these three amino-acid substitutions (W131E, L132R, F186D) did not bind CED-3 zymogen (Figure 4a) and failed to rescue the csp-2(tm3077) mutant when expressed under the control of the pie-1 promoter $\left[P_{\text {pie-1 }} \mathrm{GFP}::\right.$ CSP-2B(W131E, L132R, F186D); Figure 3f]. These results suggest that association of CSP-2B with CED-3 is important for CSP-2B to protect germ cells from apoptosis.

CSP-2B inhibits specifically autoactivation of the CED-3 zymogen. As CSP-3 inhibits apoptosis by specifically blocking autoactivation of the CED-3 zymogen, we examined whether CSP-2B has a similar activity using an in vitro CED-3 autoactivation assay described earlier. ${ }^{13}$ As shown in Figure 5a (lanes 1-3), CED-3 zymogen synthesized in rabbit reticulocyte lysate and labeled with ${ }^{35} S$-methinione was slowly autoprocessed into active forms. The autoactivation of the CED-3 zymogen was inhibited by the addition of the GST-CSP-2B protein (Figure 5a, lanes 4-6) but was not affected by the addition of a similar amount of the GST-CSP2B(W131E, L132R, F186D) protein or the GST protein (Figure 5a, lanes 1-3 and 7-9). Addition of oligomeric CED-4 to the reactions expedited the activation of the CED-3 zymogen (compare lanes 1-5 and lanes 6-10 in Figure 5b). Although GST-CSP-2B completely inhibited CED-3 autoactivation (Figure 5b, lanes 11-15), it delayed but did not block the activation of CED-3 induced by oligomeric CED-4 (Figure 5b, lanes 16-20). GST-CSP-2B also failed to inhibit the catalytic activity of the active CED-3 protease (Figure 5c). Therefore, CSP-2B acts exactly like CSP-3; it complexes with the CED-3 zymogen and inhibits its autoactivation, but is unable to block CED-4-induced CED3 activation or the activity of active CED-3.

\section{Discussion}

Apoptosis is a common feature of metazoan germline development. A wild-type $C$. elegans hermaphrodite has approximately 2000 germ cells generated during its lifetime and more than half of these cells undergo apoptosis in a random fashion. ${ }^{1}$ By contrast, somatic cell deaths in $C$. elegans occur strictly based on cell lineage information and are invariant from animal to animal. ${ }^{23}$ As such, these two apoptotic processes likely will be controlled through very different regulatory pathways. Indeed, the cell death initiator EGL-1 that is critical for somatic apoptosis is totally dispensable for physiological germ cell death ${ }^{1}$ and it is unclear how germ cell deaths are initiated. Moreover, in this study, we showed that the CED-3 caspase inhibitor, CSP-3, does not affect germ cell death (Figure $3 c$ and e), providing further evidence that key regulatory components of germ cell death differ from those of somatic cell death to achieve tissue specificity. This finding also suggests that germ cells might possess an alternative CED-3 caspase inhibitor(s) to prevent inappropriate or excessive germ cell deaths.

CSP-2 is the second caspase homolog identified in $C$. elegans and shares sequence similarity to both the large and the small subunits of CED-3. ${ }^{14}$ However, unlike CSP-1, another worm caspase homolog that possesses a caspase activity, $^{14}$ CSP-2 does not display any caspase activity in vitro, which may result from its lack of the invariant pentapeptide found in active sites of all active caspases. ${ }^{15}$ Intriguingly, the carboxyl terminus of CSP-2 is $92 \%$ identical to

Figure 2 Inactivation of csp-2 does not affect apoptosis in somatic cells. (a, b) Embryonic cell corpse assays. Cell corpses were scored in the indicated strains. The ced6(n2095) allele was used in (b). Bean (B/C), 1.5-fold (1.5), 2-fold (2), 2.5-fold (2.5), 3-fold (3), and 4-fold (4) stage embryos were scored. The $y$-axis represents the average number of cell corpses scored. Error bars are S.E.M. At least 15 embryos were scored for each stage. The significance of differences between different genetic backgrounds was determined by unpaired $t$-tests. ${ }^{\star} P<0.05 ;{ }^{* *} P<0.0001$. (c) Inactivation of csp-2 does not cause ectopic death of touch receptor neurons. An integrated transgene (bzls8) was used to monitor the survival of six touch receptor neurons (green circles) as described in 'Materials and Methods'. The percentages of animals missing one or more touch cells are shown. At least 100 animals were scored for each strain 

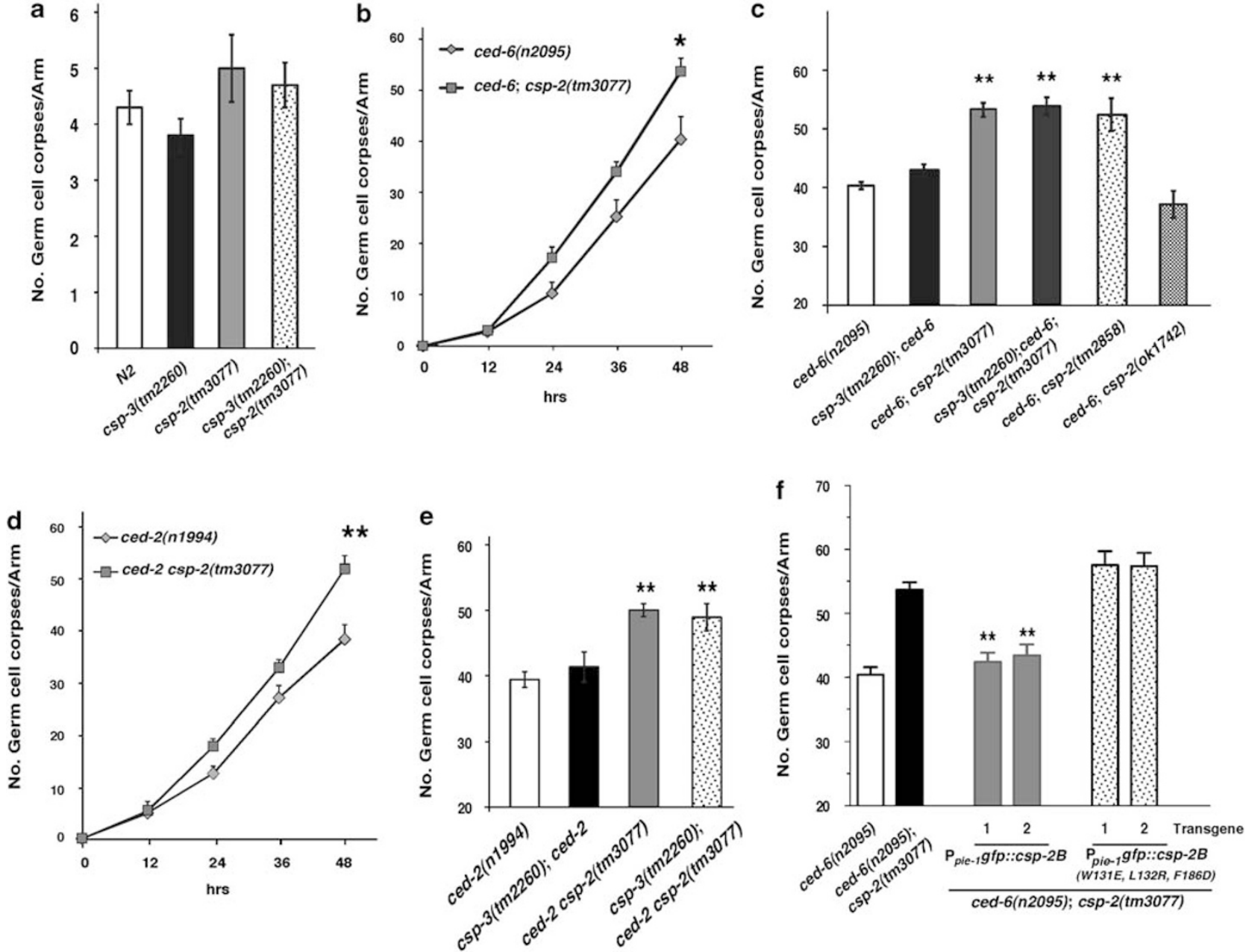

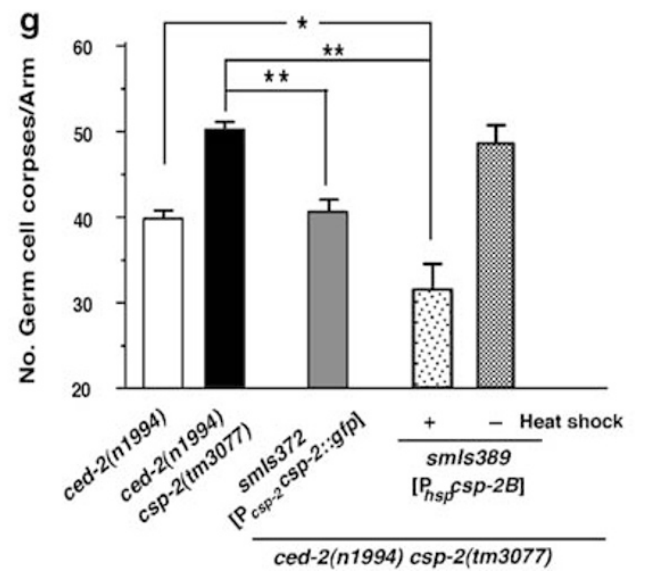

h

Genotype
bz/s 8

csp-3(tm2260); bzls 8

csp-3(tm2260); bzls8; Ex[Phspcsp-2B] \#1

csp-3(tm2260); bzls8; Ex[Phspcsp-2B] \#2

csp-3(tm2260); bzls8; Ex[P $\left.P_{h s p} c s p-2 B\right] \# 3$
Animals missing at least one touch cell (\%)

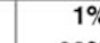

$30 \%$

$3 \%$

$0 \%$

$7 \%$

Figure 3 Loss of csp-2 causes increased apoptosis in germ cells. Germ cell corpses were scored in the indicated strains from one gonad arm $48 \mathrm{~h}$ after L4 to adult molt $(\mathbf{a}, \mathbf{c}, \mathbf{e}, \mathbf{f}$, and $\mathbf{g})$ or every $12 \mathrm{~h}$ after $\mathrm{L} 4$ to adult molt $(\mathbf{b}, \mathbf{d})$. Average numbers of germ cell corpses are shown. Error bars represent the S.E.M. At least 15 animals were scored in each strain or time point. In (b), the significance of difference between ced-6(n2095) and csp-2(tm3077); ced-6(n2095) animals was determined by two-way analysis of variance (ANOVA). ${ }^{*} P<0.05$ for the whole time course. In (c), the significance of differences between various csp; ced-6(n2095) strains and the ced-6(n2095) strain was determined by unpaired $t$-tests. ${ }^{*} P<0.0001$. All other points had $P$-values $>0.05$. In (d), the significance of difference between ced-2(n1994) and ced-2(n1994) csp2(tm3077) animals was determined by two-way ANOVA. ${ }^{*} P<0.0001$ for the whole time course. In (e), the significance of differences between different $c s p ;$ ced-2(n1994) strains and the ced-2(n1994) strain was determined by unpaired $t$-tests. ${ }^{* *} P<0.0001$. All other points had $P$-values $>0.05$. (f, $\left.\mathbf{g}\right)$ Rescue of the csp-2 mutant by $\mathrm{P}_{\text {pie-1 }}$ gfp:: csp-2B, $\mathrm{P}_{c s p-2} c s p-2::$ gfp, or $\mathrm{P}_{h s p} c s p-2 B$ transgenes. The significance of differences between transgenic strains and the ced-6(n2095); csp-2(tm3077) strain (f), between transgenic strains and the ced-2(n1994) csp-2(tm3077) strain (g), or between the sm/s389-containing strains and the ced-2(n1994) strain (g) was determined by unpaired $t$-tests. ${ }^{* *} P<0.0001 ;{ }^{*} P<0.01$. All other points had $P$-values $>0.05$. (h) The $\mathrm{P}_{h s p} c s p-2 B$ transgenes rescue the missing cell defect of the $c s p$-3(tm2260) mutant. The presence of touch cells was scored as described in Figure $2 \mathrm{C}$ 
Table 1 The csp-2 mutations cause reduced brood size

\begin{tabular}{lc}
\hline Genotype & Brood size $^{\mathrm{a}}$ \\
\hline N2 & $249 \pm 28$ \\
csp-2(tm3077) & $142 \pm 31^{\mathrm{b}}$ \\
csp-2(tm2858) & $155 \pm 19^{\mathrm{b}}$ \\
csp-2(ok1742) & $225 \pm 27$ \\
ced-9(n1653ts) & $22 \pm 11$ \\
ced-9(n1653ts); csp-2(tm3077) & $0.0 \pm 0.4^{\mathrm{c}}$ \\
\hline
\end{tabular}

${ }^{\text {a }}$ All strains were maintained at $20^{\circ} \mathrm{C}$, except that ced-9(n1653ts) and ced9(n1653ts); csp-2(tm3077) strains were maintained at $25^{\circ} \mathrm{C}$. Brood size was scored as the number of eggs laid by each hermaphrodite animal. Data shown are mean \pm S.D. and are derived from three independent experiments. Twenty animals were scored in each experiment. ${ }^{\mathrm{b}} \mathrm{Compared}$ with N2 animals, $P<0.0001$ (two-tail Student's $t$-test). Compared with csp-2(ok1742) animals, $P<0.0001$ (two-tail Student's $t$-test). ${ }^{\circ}$ Compared with ced-9(n1653ts) animals, $P<0.001$ (two-tail Student's $t$-test).

CSP-3. Phylogenetic analysis of C. elegans CED-3 and CSP proteins suggests that $c s p-2$ and $c s p-3$ may have evolved from a common ancestor (Supplementary Figure 1). Therefore, CSP-2 may behave like CSP-3 in binding to CED-3 and inhibiting CED-3 autoactivation. ${ }^{13}$ However, inactivation of csp-2 does not seem to affect somatic cell death, nor does it enhance the csp-3(If) defect in somatic cells (Figure 2), indicating that $c s p-2$ does not affect apoptosis in somatic cells. On the other hand, inactivation of csp-2 does increase apoptosis in germ cells (Figure 3), which is not affected by loss of csp-3, suggesting that CSP-2, but not CSP-3, acts specifically in germ cells to inhibit apoptosis. Consistent with these findings, CSP-2 is specifically expressed in the $C$. elegans germline. Therefore, CSP-2 and CSP-3, two closely related paralogs, somehow diverged during evolution to act in germ cells and somatic cells, respectively. This change created tissue-specific cell death regulation.

Given the unique mode by which CSP-3 regulates CED-3 autoactivation and somatic apoptosis, ${ }^{13}$ we tested how CSP-2 inhibits cell death. Our biochemical analysis indicates that CSP-2 directly associates with the CED-3 zymogen through both its small and large subunits (Figure $4 a$ and $b$ ). Three amino-acid substitutions in CSP-2 (W131E, L132R, F186D) almost completely abolish the binding of CSP-2 to the CED-3 zymogen in vitro (Figure 4a) and the apoptosis inhibitory activity of CSP-2 in vivo (Figure $3 \mathrm{f}$ ), suggesting that binding of CSP-2 to CED-3 is critical for its anti-apoptosis function. Moreover, we found that CSP-2 acts exactly like CSP-3 in regulating the activity of CED-3: it associates with the CED-3 zymogen and inhibits its autoactivation, but is unable to block oligomeric CED-4-induced CED-3 activation or inhibit the catalytic activity of activated CED-3 protease (Figure $5 a-C$ ). These results establish CSP-2 as the second caspase inhibitor in C. elegans that acts specifically to inhibit autoactivation of CED-3 in germ cells, thereby preventing inappropriate germ cell deaths.

It is interesting that two different caspase homologs use the same strategy to inhibit CED-3 activation and apoptosis in two different tissues, somatic cells and germ cells (Figure $5 \mathrm{~d}$ ). The repeated use of this unique strategy to negatively regulate CED-3 caspase activation underscores the importance of keeping caspases in check in living cells. In the absence of other obvious caspase inhibitors such as IAPs in C. elegans, such caspase-like inhibitors are important players in modulating the level of CED-3 activation and preventing inadvertent CED-3 activation in cells that should live, without interfering with normal cell death induced by CED-4 (Figure $5 d$ ), as CSP2B only delayed but did not block CED-4-induced CED-3 activation (Figure $5 b$ ). We propose that similar incomplete or inactive caspase homologs exist in other organisms and could use the same mechanism to negatively regulate caspase activation and apoptosis.

\section{Materials and Methods}

C. elegans strains. We cultured strains of $C$. elegans at $20^{\circ} \mathrm{C}$ using standard protocols. ${ }^{24}$ The Bristol strain N2 was used as the wild-type stain. Most of the alleles including ced-2(n1994) and ced-6(n2095) used in this study have been described earlier ${ }^{25}$ except csp-2(tm3077), csp-2(tm2858), csp-2(ok1742), and bzls8. The csp2(ok1742) mutant was obtained from Caenorhabditis Genetics Center (CGC) and described in Wormbase (http://www.wormbase.org/). bz/s8 is an integrated transgene located on $L G X$ and contains a $P_{m e c-4 g f p}$ construct, ${ }^{26}$ which directs GFP expression in six $C$. elegans touch receptor neurons. All strains were backcrossed with N2 animals 4-10 times before analysis.

Quantification of germline apoptosis. We identified and quantified germ cell corpses based on their characteristic morphology when viewed with Nomarski optics as described earlier. ${ }^{1}$ Germline apoptosis was assessed in staged adult animals. At least 15 animals were scored for each time point. Data are reported as mean number of germline cell corpses \pm standard error of the mean (S.E.M).

Isolation of csp-2 (tm3077) and csp-2(tm2858) deletion alleles. We isolated the csp-2(tm3077) and csp-2(tm2858) deletion alleles from TMP/UV mutagenized worms. ${ }^{27}$ Nested primers used to screen for the csp-2(tm3077) and csp-2(tm2858) alleles by PCR were $5^{\prime}$ GCCGGGCTATCATAATTAAC $3^{\prime}$ and $5^{\prime}$ ATACTGATCACCGAGGCCAT $3^{\prime}$ for the first round amplification and $5^{\prime}$ ACGTTTGGGATATCAGTCGA' and 5' CACGTCATTTCTAGACGTCG $3^{\prime}$ for the second round amplification. Both mutants were backcrossed with wild-type (N2) animals at least four times before they were analyzed further.

RNA isolation and RT-PCR analysis of csp-2 transcripts. We isolated poly $(A) n$ mRNA from mix-stage wild-type hermaphrodite animals treated with TRIzol reagent (Invitrogen, Carlsbad, CA, USA). First-strand cDNA was reverse transcribed by using Superscript III kit (Invitrogen). PCR was performed using specific primers (csp-2A, $5^{\prime}$ GAGCAGTATAGTGCGTTGAGA-GAG $3^{\prime}$ and $5^{\prime}$ CTTCCTCTTCCCTTTCTCTCTGTT $3^{\prime}$; csp-2B, $5^{\prime}$ ACGTTTGGGA-TATCAGTCGA $3^{\prime}$ and $5^{\prime}$ CTAGACGTCGAAGAATAGTTG $3^{\prime}$ ), which produce a $706 \mathrm{bp}$ (csp-2A) and a $622 \mathrm{bp}(\mathrm{csp}-2 \mathrm{~B}) \mathrm{cDNA}$ fragment, respectively. The PCR products were resolved on a $1.5 \%$ agarose gel.

Quantification of somatic apoptotic cell corpses and cells labeled with GFP. We counted cell corpses in animals at various embryonic and larval stages using Nomarski optics as described earlier. ${ }^{13}$ In addition, we quantified touch receptor neuron cells in bzls8 as described earlier. ${ }^{13}$

Plasmids and transgenic strains. We generated the $\mathrm{P}_{\text {csp-2 }}$ csp-2::gfp fusion construct by inserting a csp-2 genomic fragment containing $4175 \mathrm{bp}$ sequence upstream of the CSP-2A initiation ATG codon and the whole csp-2 coding region into the pPD95.79 vector. The resulting plasmid was introduced into ced1(e1735); unc-119(ed3) by the microparticle bombardment together with a marker plasmid MM016B that contains the wild-type unc-119 gene to obtain a low-copy integrated array sm/s37219. To examine the expression patterns of CSP-2::GFP, anti-GFP immunostaining was carried out on the exposed gonads and embryos of smls372 animals. ${ }^{28}$

We also generated the $\mathrm{P}_{\text {pie-1 }} \mathrm{GFP}:: \mathrm{CSP}-2 \mathrm{~B}$ fusion construct by inserting the fulllength $c s p-2 B$ cDNA into pTE5 vector $\left(P_{\text {pie-1 }} \mathrm{GFP}\right)$ through Spel and Apal site. The $\mathrm{P}_{\text {pie-1 }}$ GFP :: CSP-2B fusion construct (wild type or mutant with W131E, L132R, F186D substitutions) was introduced into the ced-6(n2095); csp-2(tm3077) animals through complex DNA arrays as described earlier ${ }^{29}$ and examined for rescue of increased germ cell death phenotype. 

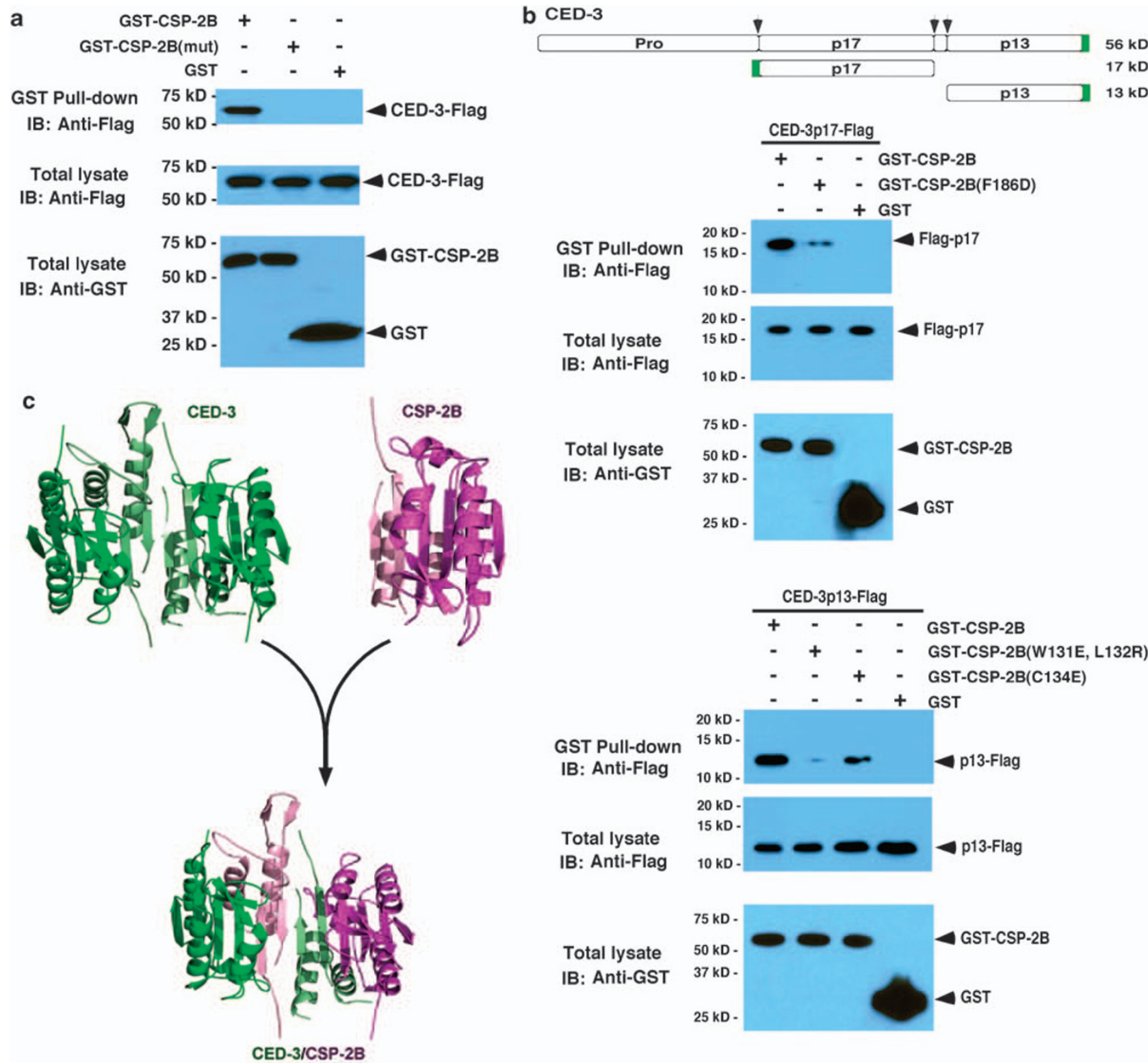

Figure 4 CSP-2B associates with CED-3 in vitro. (a) CSP-2B binds to the CED-3 zymogen. GST-CSP-2B, GST-CSP-2B(mut), or GST was co-expressed in bacteria with the CED-3 zymogen tagged with a Flag epitode (CED-3-Flag). 'mut' stands for W131E, L132R, and F186D substitutions. One portion of the soluble fraction was used in the western blot analysis to examine the expression levels of GST fusion proteins and CED-3-Flag using anti-GST and anti-Flag antibodies, respectively. The remaining portion of the soluble fraction was used in the GST fusion protein pull-down experiment and the amount of CED-3-Flag pulled down was analyzed by the western blot analysis using an anti-Flag antibody. (b) CSP-2B associates with both the large subunit and the small subunit of the CED-3 zymogen in vitro. The cartoon shows the domain structure of the CED-3 zymogen, with arrows indicating the three proteolytic cleavage sites that lead to the activation of the CED-3 zymogen. The large (p17) and the small ( $p 13)$ subunits of CED-3 are shown below as boxes. The Flag epitode is labeled with green. GST-CSP-2B, GST-CSP-2B(F186D), GST-CSP-2B(W131E, L132R), GST-CSP-2B(C134E), or GST was co-expressed in bacteria with the CED-3 large subunit (Flag-p17) or the small subunit (p13-Flag), both of which are tagged with a Flag epitode. One portion of the soluble fraction was used in the western blot analysis to examine the expression levels of GST fusion proteins and CED-3 subunits using anti-GST and anti-Flag antibodies, respectively. The remaining portion of the soluble fraction was used in the GST fusion protein pull-down experiments and the amount of CED- 3 subunits pulled down was analyzed by the western blot analysis using an anti-Flag antibody. (c) Structural model of the CED-3/CSP-2B complex. As all caspases with known structures share a highly conserved core structure, the structure of caspase-3 (PDB code 1pau) was used to represent the structures of CED-3 and CSP-2B. The large subunit of CED-3 is shown in green, the small subunit shown in lemon. The large subunit of CSP-2B is shown in magenta, the small subunit in light pink

Expression and purification of the CSP-2B and CED-3 proteins. We generated all protein expression constructs using the standard PCR-based cloning strategy and verified the clones through sequencing. CSP-2B and CED-3 proteins were expressed either individually or together in Escherichia coli strain BL21(DE3) as an N-terminally GST fusion protein and a C-terminally Flag-tagged protein using a pET-41b vector and a pET-3a vector (Novagen, Schwallbach, Germany), respectively. The soluble fraction of the $E$. coll lysate expressing GST-CSP-2B proteins was purified using a Glutathione Sepharose column and eluted with $10 \mathrm{mM}$ reduced glutathione (Amersham, Piscataway, NJ, USA).

GST fusion protein pull-down assays. The GST-CSP-2B fusion protein (wild type or mutants) or GST was co-expressed with CED-3-Flag, CED-3p13-Flag, or Flag-CED-3p17 in BL21(DE3). Bacteria were lysed by sonication in the lysis buffer 
a

\begin{tabular}{|c|c|c|}
\hline+ & - & - \\
\hline- & + & - \\
\hline- & - & + \\
\hline
\end{tabular}

GST

GST-CSP-2B

GST-CSP-2B(mut)

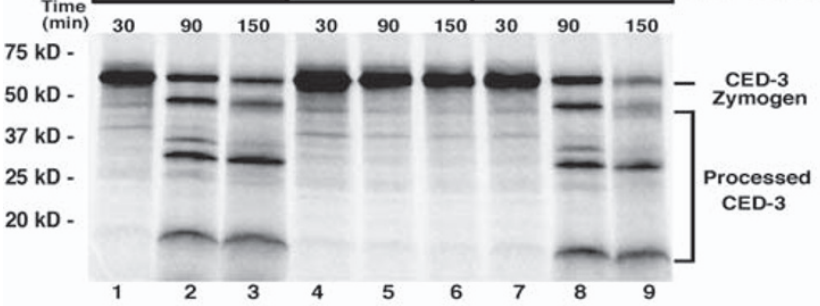

C

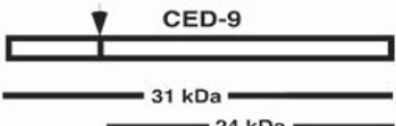

\begin{tabular}{|c|c|c|c|c|}
\cline { 2 - 4 } acCED-3 & - & + & + & + \\
\cline { 2 - 5 } $\mathrm{CH}_{3}$ COOI & - & - & - & + \\
\cline { 2 - 5 } GST & - & + & - & + \\
\cline { 2 - 5 } GST-CSP-2B & - & - & + & - \\
\cline { 2 - 5 } & &
\end{tabular}

$37 \mathrm{kD}$ -

$25 \mathrm{kD}$ -

$20 \mathrm{kD}$ -

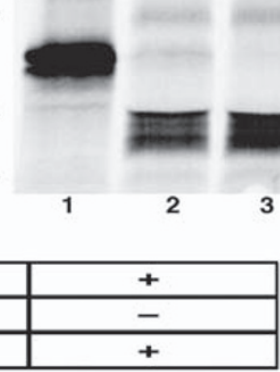

\begin{tabular}{|c|c|c|c|c|}
\hline GST & + & + & - & - \\
\hline GST-CSP-2B & - & - & + & + \\
\hline
\end{tabular}

$\begin{array}{llllllllllllllllllllll}(\min ) & 30 & 80 & 130 & 180 & 230 & 30 & 80 & 130 & 180 & 230 & 30 & 80 & 130 & 180 & 230 & 30 & 80 & 130 & 180 & 230 & \end{array}$

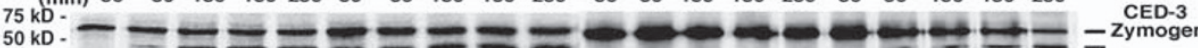

$37 \mathrm{kD}$.

$20 \mathrm{kD}$ -
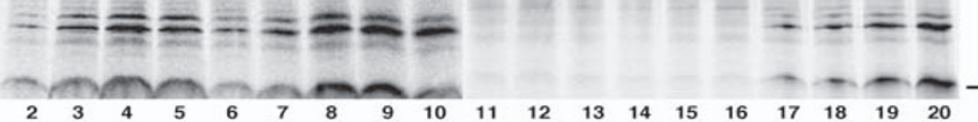

rocessed

CED-3 d

\section{Somatic cells}

CED-3

Zymogen

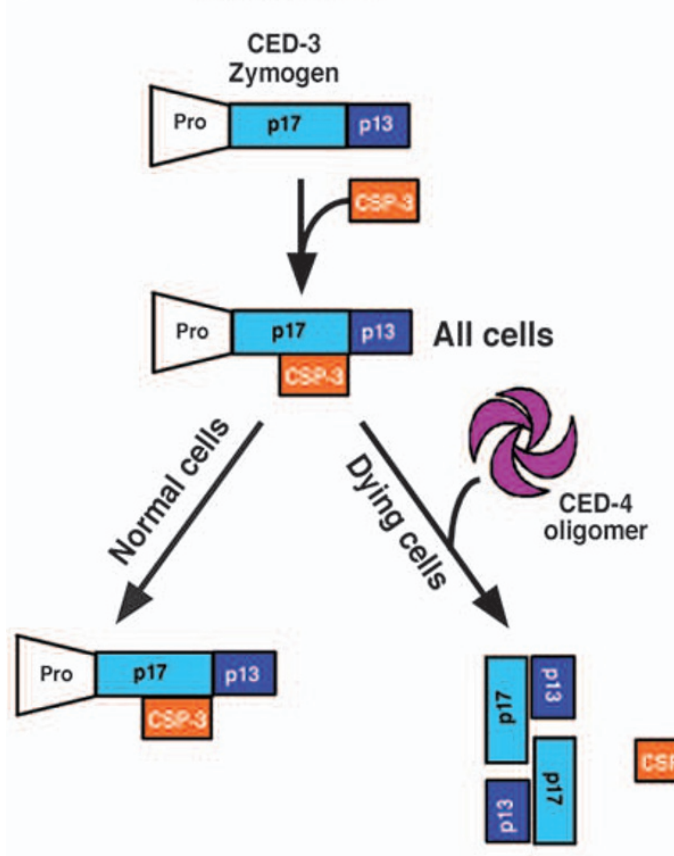

acCED-3

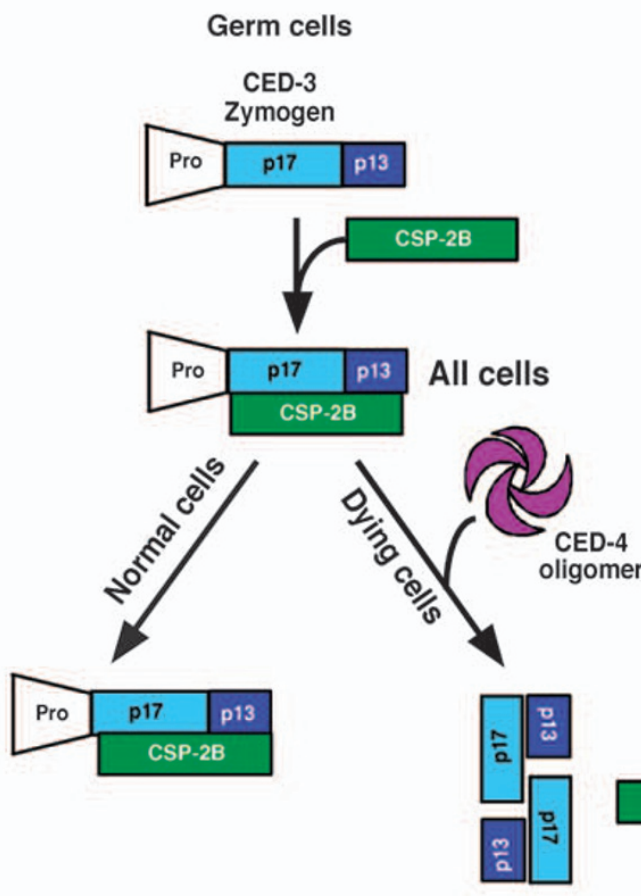

CSP-2B
CED-9

-31 kDa

-24 kDa

Figure 5 CSP-2B specifically inhibits the autoactivation of the CED-3 zymogen in vitro. (a) CSP-2B inhibits autoactivation of the CED-3 zymogen. GST (93 nM, lanes 1-3), GST-CSP-2B (43 nM, lanes 4-6), or GST-CSP-2B(mut) (43 nM, lanes 7-9) were incubated with ${ }^{35}$ S-methionine-labeled CED-3 zymogen as described in 'Materials and Methods'. At different time points (30,90, and $150 \mathrm{~min}$ ), an aliquot of the reaction was taken out and SDS sampling buffer was added to stop the reaction. The samples were resolved by $15 \%$ SDS polyacrylamide gel electrophoresis (SDS-PAGE) and subjected to autoradiography. (b) CSP-2B delays but does not block CED-4-mediated activation of CED-3. GST $\left(93 \mathrm{nM}\right.$ ) or GST-CSP-2B ( $43 \mathrm{nM}$ ) was incubated with ${ }^{35} \mathrm{~S}$-methionine-labeled CED-3 zymogen in the absence or presence of oligomeric CED-4 (40 nM) (added $20 \mathrm{~min}$ later). At 30, 80, 130, 180, and $230 \mathrm{~min}$, an aliquot of the reaction was taken out and SDS sampling buffer was added. The samples were resolved by $15 \%$ SDS-PAGE and subjected to autoradiography. (c) CSP-2B does not inhibit the activity of the active CED-3 protease in vitro. CED-3-Flag was co-expressed with GST or GST-CSP-2B for $3 \mathrm{~h}$ in bacteria. The bacterial lysate containing similar levels of active CED-3 (acCED-3) and GST fusion proteins was incubated with ${ }^{35} \mathrm{~S}$-methionine-labeled CED-9 for $2 \mathrm{~h}$ at $30^{\circ} \mathrm{C}$. In lane 4 , the caspase inhibitor, iodoacetic acid $(5 \mathrm{mM})$, was included. The reactions were resolved by $15 \%$ SDS-PAGE and detected by autoradiography. (d) A working model of how CSP-3 (left panel) and CSP-2B (right panel) inhibit CED-3 autoactivation and apoptosis in C. elegans somatic and germ cells, respectively 
[50 mM Tris at pH 8.0, 0.5 mM EDTA, $150 \mathrm{mM} \mathrm{NaCl}, 0.01 \%$ (v/v) Triton X-100, and $0.5 \mathrm{mM}$ sucrose] with protease inhibitors and the soluble fraction was incubated with Glutathione Sepharose beads at $4{ }^{\circ} \mathrm{C}$ for $2 \mathrm{~h}$. The Sepharose beads were then washed five times with the same buffer before the proteins were resolved on a $15 \%$ SDS polyacrylamide gel (SDS-PAGE), transferred to a PVDF membrane, and detected by immunoblotting with an anti-Flag antibody (Sigma, St Louis, MO, USA).

In vitro CED-3 zymogen activation assay. The CED-3 zymogen was first synthesized and labeled with ${ }^{35} \mathrm{~S}$-methionine in the TNT transcription/translation coupled system (Promega, Madison, WI, USA) at $30^{\circ} \mathrm{C}$ as described EARLIER, ${ }^{30}$ in the presence of equal amount of GST-CSP-2B, GST-CSP$2 \mathrm{~B}(\mathrm{~W} 131 \mathrm{E}, \mathrm{L} 132 \mathrm{R}, \mathrm{F} 186 \mathrm{D})$, or GST. An aliquot of the reaction was taken out at different time points and mixed with SDS sampling buffer to stop the reaction. For CED-4-mediated CED-3 activation assay, oligomeric CED-4 was added $20 \mathrm{~min}$ after the initiation of the translation reaction. An aliquot of the reaction was then taken out at different time points and mixed with SDS sampling buffer to stop the reaction. All samples were resolved by $15 \%$ SDS-PAGE and analyzed by autoradiography.

Statistics. We performed all statistical analysis using Prism (GraphPad Software). All error bars indicate S.E.M. All $t$-tests are two-tailed unpaired $t$-tests. Time courses curves were analyzed by two-way ANOVA.

Acknowledgements. We thank $N$ Pace for advice on phylogenetic analysis, $Y$ Shi and members of the Xue laboratory for comments and discussions, L Yang, HW Yang, and CL Sun for technical support, S Shaham for cDNA clones, and M Driscoll (Rutgers University) for the bzls8 strain. This work was supported by NIH R01 grants (GM059083 and GM079097) and a Burroughs Welcome Fund Award to DX and a grant from MEXT of Japan to SM. XG was supported by U Colorado Matching Grant to the SCR Training Grant (T32 GM08759).

1. Gumienny TL, Lambie E, Hartwieg E, Horvitz HR, Hengartner MO. Genetic control of programmed cell death in the Caenorhabditis elegans hermaphrodite germline. Development 1999; 126: 1011-1022.

2. Gartner A, Boag PR, Blackwell TK. Germline survival and apoptosis. WormBook 2008: 1-20. doi/10.1895/wormbook.1.145.1, http://www.nature.com/cdd.

3. Andux S, Ellis RE. Apoptosis maintains oocyte quality in aging Caenorhabditis elegans females. PLOS Genet 2008; 4: e1000295.

4. Gartner A, Milstein S, Ahmed S, Hodgkin J, Hengartner MO. A conserved checkpoint pathway mediates DNA damage - induced apoptosis and cell cycle arrest in $C$. elegans. Mol Cell 2000; 5: 435-443.

5. Shi Y. Mechanisms of caspase activation and inhibition during apoptosis. Mol Cell 2002; 9: 459-470.

6. Boatright KM, Salvesen GS. Mechanisms of caspase activation. Curr Opin Cell Biol 2003; 15: 725-731.

7. Deveraux QL, Reed JC. IAP family proteins - suppressors of apoptosis. Genes Dev 1999; 13: $239-252$.

8. Deveraux QL, Takahashi R, Salvesen GS, Reed JC. X-linked IAP is a direct inhibitor of cell-death proteases. Nature 1997; 388: 300-304
9. Roy N, Deveraux QL, Takahashi R, Salvesen GS, Reed JC. The c-IAP-1 and C-IAP-2 proteins are direct inhibitors of specific caspases. EMBO J 1997; 16: 6914-6925.

10. Deveraux QL, Roy N, Stennicke HR, Van Arsdale T, Zhou Q, Srinivasula SM et al. IAPs block apoptotic events induced by caspase- 8 and cytochrome $c$ by direct inhibition of distinct caspases. EMBO J 1998; 17: 2215-2223.

11. Meier $P$, Silke J, Leevers SJ, Evan GI. The Drosophila caspase DRONC is regulated by DIAP1. EMBO J 2000; 19: 598-611.

12. Hawkins CJ, Wang SL, Hay BA. A cloning method to identify caspases and their regulators in yeast: identification of Drosophila IAP1 as an inhibitor of the Drosophila caspase DCP-1. Proc Natl Acad Sci USA 1999; 96: 2885-2890.

13. Geng $X$, Shi $Y$, Nakagawa A, Yoshina S, Mitani S, Xue D. Inhibition of CED-3 zymogen activation and apoptosis in Caenorhabditis elegans by caspase homolog CSP-3. Nat Struct Mol Biol 2008; 15: 1094-1101.

14. Shaham S. Identification of multiple Caenorhabditis elegans caspases and their potentia roles in proteolytic cascades. J Biol Chem 1998; 273: 35109-35117.

15. Cohen GM. Caspases: the executioners of apoptosis. Biochem J 1997; 326: 1-16

16. Blumenthal T. Trans-splicing and operons. WormBook 2005: 1-9.

17. Ellis RE, Jacobson DM, Horvitz HR. Genes required for the engulfment of cell corpses during programmed cell death in Caenorhabditis elegans. Genetics 1991; 129: 79-94.

18. Hengartner MO, Ellis RE, Horvitz HR. Caenorhabditis elegans gene ced-9 protects cells from programmed cell death. Nature 1992; 356: 494-499.

19. Praitis V, Casey E, Collar D, Austin J. Creation of low-copy integrated transgenic lines in Caenorhabditis elegans. Genetics 2001; 157: 1217-1226.

20. Tenenhaus C, Subramaniam K, Dunn MA, Seydoux G. PIE-1 is a bifunctional protein that regulates maternal and zygotic gene expression in the embryonic germ line of Caenorhabditis elegans. Genes Dev 2001; 15: 1031-1040.

21. Xue D, Shaham S, Horvitz HR. The Caenorhabditis elegans cell-death protein CED-3 is a cysteine protease with substrate specificities similar to those of the human CPP32 protease. Genes Dev 1996; 10: 1073-1083.

22. Rotonda J, Nicholson DW, Fazil KM, Gallant M, Gareau Y, Labelle M et al. The threedimensional structure of apopain/CPP32, a key mediator of apoptosis. Nat Struct Biol 1996; 3: 619-625

23. Sulston JE, Horvitz HR. Post-embryonic cell lineages of the nematode, Caenorhabditis elegans. Dev Biol 1977; 56: 110-156.

24. Brenner S. The genetics of Caenorhabditis elegans. Genetics 1974; 77: 71-94.

25. Riddle DL, Blumenthal T, Meyer BJ, Preiss JR (eds). C. elegans II. Cold Spring Harbor Laboratory Press: Cold Spring Harbor, NY, 1997.

26. Harbinder S, Tavernarakis N, Herndon LA, Kinnell M, Xu SQ, Fire A et al. Genetically targeted cell disruption in Caenorhabditis elegans. Proc Natl Acad Sci USA 1997; 94: 13128-13133.

27. Gengyo-Ando K, Mitani S. Characterization of mutations induced by ethyl methanesulfonate, UV, and trimethylpsoralen in the nematode Caenorhabditis elegans. Biochem Biophys Res Commun 2000; 269: 64-69.

28. Darland-Ransom M, Wang X, Sun CL, Mapes J, Gengyo-Ando K, Mitani S et al. Role of $C$. elegans TAT-1 protein in maintaining plasma membrane phosphatidylserine asymmetry. Science 2008; 320: 528-531.

29. Wang X, Wang J, Gengyo-Ando K, Gu L, Sun CL, Yang C et al. C. elegans mitochondria factor WAH-1 promotes phosphatidylserine externalization in apoptotic cells through phospholipid scramblase SCRM-1. Nat Cell Biol 2007; 9: 541-549.

30. Yan N, Chai J, Lee ES, Gu L, Liu Q, He J et al. Structure of the CED-4-CED-9 complex provides insights into programmed cell death in Caenorhabditis elegans. Nature 2005; 437: 831-837.

\section{Supplementary Information accompanies the paper on Cell Death and Differentiation website (http://www.nature.com/cdd)}

